\title{
COINVOLGIMENTO LAVORATIVO DEGLI EDUCATORI DEL NIDO E DEGLI INSEGNANTI DELLA SCUOLA DELL'INFANZIA: STUDIO QUALI-QUANTITATIVO.
}

\author{
Simona De Stasio \\ Francesca Boldrini \\ Benedetta Ragni \\ Chiara Bacile \\ Eleonora Tacconelli \\ Paula Benevene \\ Libera Università Maria SS. Assunta \\ Dipartimento di Scienze Umane, Roma \\ s.destasio@lumsa.it
}

Fecha de Recepción: 5 Septiembre 2018

Fecha de Admisión: 1 Octubre 2018

\section{RIASUNTO}

La letteratura mostra come il coinvolgimento lavorativo, l'entusiasmo e il vigore dei docenti siano predittivi delle prestazioni lavorative ed abbiano un effetto benefico sulle relazioni positive insegnante-studente e sugli esiti accademici degli studenti (Bakker, Schaufeli, Leiter \& Taris, 2008; Bakker, Van Emmerik \& Euwema, 2006; Duckworth, Quinn \& Seligman, 2009). L'obiettivo del presente studio di carattere quali-quantitativo è stato duplice: 1) valutare il ruolo predittivo, sul coinvolgimento lavorativo degli operatori dei servizi educativi e scolastici $0 / 6$ anni, di variabili disposizionali (benessere soggettivo, compassione, affettività disposizionale positiva) e di natura socio-contestuale (strategie proattive auto ed etero regolative, clima costruttivo); 2) esplorare sul piano qualitativo le dimensioni di promozione del benessere nei servizi. Ad un gruppo di 189 educatori e insegnanti della scuola dell'infanzia sono stati somministrati I'UWES-17 (Schaufeli \& Bakker, 2004), la Santa Clara Brief Compassion Scale (Hwang, Plante, \& Lackey, 2008), la Proactive Strategy Scale (Salmela-Aro, 2009), la Positive and Negative Affect Schedule (Watson, Clark, \& Tellegen, 1988), la Subjective Happiness Scale (Lyuborminsky \& Lepper, 1999) la Teacher selfefficacy ed un intervista qualitativa costruita ad hoc sugli aspetti di promozione del benessere. I principali esiti quantitativi evidenziano come a parità di benessere soggettivo e di affettività disposizionale positiva, la compassione e le strategie proattive sono predittori di un maggior coinvolgimento lavorativo.

Parole chiave: engagement, educatori del nido, insegnanti della scuola dell'infanzia, compassione, strategie proattive, benessere soggettivo. 


\begin{abstract}
Research shows as work-engagement, enthusiasm and vigor predict work performance and have positive effects on teacher-student relationships and on students' academic outcomes (Bakker, Schaufeli, Leiter \& Taris, 2008; Bakker, Van Emmerik \& Euwema, 2006; Duckworth, Quinn \& Seligman, 2009). According to our quali-quantitative study, two main aims were set: 1) the evaluation of the predictive role of some dispositional variables (subjective wellbeing, compassion and dispositional positive affect) and of some socio-contextual variables (self/co-regulated proactive strategies, constructive climate) on teachers' work-engagement in educational and scholastic services $0 / 6$ years; 2) the qualitatively exploration of dimensions of promoting well-being in educational services. A group of 189 educators and kindergarten teachers was asked to fill the following questionnaires: the UWES-17 (Schaufeli, 2003), the Santa Clara Brief Compassion Scale (Sprecher \& Fehr, 2005), the Proactive Strategy Scale (Salmela-Aro, 2009), the Positive and Negative Affect Schedule (Watson et al, 1988), the Subjective Happiness Scale (Lyuborminsky \& Lepper, 1999) and the Teacher self-efficacy and ad hoc interview on components of promoting well-being in educational services. Our main quantitative outcomes showed that compassion and proactive strategies predict higher levels of work-engagement over subjective well-being and dispositional positive affect.
\end{abstract}

Keywords: engagement, nest educators, kindergarten teachers, compassion, proactive strategies, subjective well-being.

\title{
INTRODUZIONE
}

Diversi studi si sono focalizzati sull'importanza della dimensione relazionale (Maslach et al., 1999; Gwede, Johnson, Roberts, \& Cantor, 2005; Albanese, Girani, De Marco, \& Fiorilli, 2009; Fiorilli, De Stasio, Benevene, lezzi, Pepe, \& Albanese 2015) nel lavoro di docenti sottolineando come gli insegnanti vivano quotidianamente interazioni ad elevato coinvolgimento emotivo, la cui qualità rappresenta un fattore di protezione (Saarni, 1999) per la loro soddisfazione lavorativa e il loro benessere (Albanese, Pepe, Fiorilli, Gabola, 2014; Fiorilli et al., 2015). La qualità dell'esperienza lavorativa degli insegnanti inoltre, risulta essere influenzata anche da tratti disposizionali individuali come il livello di benessere soggettivo percepito e quello di compassione nei confronti degli altri (Boehm \& Lyubomirsky, 2008; Burger \& Caldwell, 2000; Lilius et al., 2008; Morgeson \& Humphrey, 2006; Warr, 2007). Studi precedenti mostrano come il sentirsi valorizzati e oggetto di attenzioni da parte di colleghi e dirigenti aumenti l'investimento e la soddisfazione lavorativa dei docenti a scuola (Baumeister \& Leary 1995; Eldor \& Shoshani, 2016). La letteratura ci mostra inoltre come la presenza di un'affettività disposizionale positiva, come forma di regolazione emotiva, fornisca agli insegnanti maggiori risorse per fronteggiare lo stress e le sfide impegnative a scuola (Tugade \& Fredrickson, 2004), prevenendo forme di grave disagio come la sindrome di burnout (Brackett, Palomera, Mojsa-Kaja, Reyes, \& Salovey, 2010). Alla luce delle considerazioni fin qui trattate, è sembrato interessante approfondire il ruolo predittivo di variabili riconducibili a risorse individuali quali la compassione, il benessere soggettivo e l'affettività positiva e di variabili di natura sociocontestuale quali le strategie proattive e l'autoefficacia collettiva, sul coinvolgimento lavorativo degli operatori dei servizi educativi e scolastici $0 / 6$ anni, contesto scolastico ad oggi scarsamente indagato rispetto al tema del benessere degli educatori e degli insegnanti. A partire dai lavori di Fredrickson (2001) ci proponiamo di estendere la sua "Broaden-and-Build Theory of positive emotions" al contesto scolastico mostrando come esperire frequentemente affetti positivi da parte 
degli insegnanti, in relazione ai loro livelli di benessere soggettivo e di compassione a lavoro, possa essere associato ad un più elevato coinvolgimento lavorativo. In accordo con questa teoria, infatti, sperimentare emozioni positive a lavoro contribuirebbe ad espandere ed allargare il repertorio di pensieri e azioni degli individui e rafforzerebbe allo stesso tempo le loro risorse individuali (Fredrickson \& Joiner, 2002; Fredrickson, 2003), potenziando la loro sensibilità e la loro apertura verso gli altri (Cropanzano \& Wright, 2001; Fredrickson \& Losada, 2005; Fredrickson, 2013; Kok et al., 2013). Un'ottimale disposizione degli insegnanti verso il proprio contesto lavorativo, in termini di vigore e soddisfazione lavorativa, potrebbe essere vista come l'esito di frequenti esperienze di emozioni positive che nel tempo si accumulerebbero e consoliderebbero le loro risorse e il loro adattamento all'ambiente di lavoro (Fredrickson, 2001; Fredrickson, Tugade, Waugh, \& Larkin, 2003; Weiss \& Cropanzano, 1996).

\section{Aspetti Disposizionali e Coinvolgimento Lavorativo}

Diversi autori hanno osservato come alcuni fattori disposizionali individuali, quali il benessere soggettivo, la compassione e la presenza di un'affettività disposizionale positiva, come forma di regolazione emotiva, siano in grado di influenzare l'esperienza lavorativa (e.g., Boehm \& Lyubomirsky, 2008; Burger \& Caldwell, 2000; Lilius, Worline, Maitlis, Kanov, Dutton, \& Frost, 2008; Morgeson \& Humphrey, 2006; Warr, 2007, Tugade \& Fredrickson, 2004).

II benessere soggettivo percepito deriva da una valutazione globalmente positiva della propria vita e si riferisce a domini quali le relazioni interpersonali e in particolare il lavoro (Diener, 2000). Questa definizione, però, risulta complessa se si tiene in considerazione il fatto che alcune persone sembrerebbero definirsi felici o infelici indipendentemente dagli eventi di vita a cui fanno fronte. Furono Lyubomirsky e Lepper, nel 1999, ad introdurre il concetto di benessere soggettivo come fattore disposizionale in grado di spiegare le differenze individuali nel codificare, interpretare e rispondere emotivamente agli eventi di vita (Lyubomirsky, 2001). Come mostrato da diversi studi, infatti, il benessere soggettivo percepito influenzerebbe il modo in cui le persone si adattano alle situazioni, agli eventi e alla vita di tutti i giorni (Lucas, 2007; Luhmann et al., 2012). In accordo alla "Broaden-and-Build-Theory of positive emotions" (Fredrickson, 2001) sopra citata, il benessere soggettivo potrebbe giocare un ruolo importante nel predire il modo in cui le persone gestiscono e vivono le loro esperienze all'interno del proprio contesto lavorativo (Lyubomirsky, King, \& Diener 2005) e allo stesso tempo avere conseguenze positive sugli outcome lavorativi, associandosi a performance migliori, a maggiore successo e ad un elevato livello di supporto sociale percepito da parte dei colleghi e rivolto agli stessi (Boehm \& Lyubomirsky, 2008; Burger \& Caldwell, 2000; Staw \& Barsade, 1993; Staw, Sutton, \& Pelled, 1994). Da studi precedenti sappiamo che lavoratori più felici infatti mostrerebbero un coinvolgimento maggiore in comportamenti altruistici e cooperativi, contribuendo a migliorare il clima lavorativo generale e apportando benefici alla produttività lavorativa (Boehm \& Lyubomirsky, 2008; Borman, Penner, Allen, \& Motowidlo, 2001; llies, Scott, \& Judge, 2006; Lee \& Allen, 2002; Miles, Borman, Spector, \& Fox, 2002). Inoltre essi sembrerebbero affrontare in maniera più efficace il sovraccarico lavorativo e lo stress, prendendo decisioni migliori a lavoro (Iverson, Olekalns, \& Erwin, 1998; Morgeson \& Humphrey, 2006; Staw \& Barsade, 1993; Warr, 2007).

Per ciò che concerne la compassione, essa può essere definita come un aspetto disposizionale di natura prosociale (Hoffman, 2000), caratterizzato da cura, affetto e gentilezza verso gli altri e dalla 
tendenza a fornire loro supporto nel momento di bisogno (Sprecher \& Fear, 2005). Nonostante molti contesti lavorativi siano interessati dalla presenza di atteggiamenti e comportamenti compassionevoli (Eldor e Shishani, 2016), pochi studi hanno considerato la relazione fra questi fattori e l'esperienza lavorativa che gli individui fanno (e.g., O'Brien, 2006; Tehan \& Robinson, 2009). Lilius e colleghi (2008) hanno evidenziato come la compassione a lavoro possa essere messa in pratica attraverso diverse strategie ad esempio fornendo supporto emotivo o permettendo ai colleghi di avere orari più flessibili; inoltre la compassione a lavoro sembrerebbe avere effetti a lungo termine sulla qualità dell'esperienza lavorativa e sulla dedizione in termini affettivi (Lilius et al., 2008). L'ambiente educativo e scolastico rivolto ai bambini tra 0 e 6 anni risulta particolarmente ricco di comportamenti compassionevoli, poiché gli insegnanti sono costantemente coinvolti in relazioni di cura con i loro piccoli alunni (Sutton \& Wheatley, 2003). Va sottolineato come fino a questo momento, la compassione sia stata studiata in particolar modo nel lavoro educativo come espressione degli insegnanti verso gli studenti. In realtà, non solo gli studenti, ma anche gli altri adulti presenti nella scuola, primi fra tutti gli stessi docenti, necessitano di esprimere e ricevere affetto ed emozioni positive. Dai pochi studi effettuati a riguardo, è emerso come la compassione tra gli insegnanti possa promuovere sensazioni di connessione emotiva a lavoro e contribuire a consolidare nessi tra supporto organizzativo e investimento sul lavoro (Eldor \& Shoshani, 2016) e come possa inoltre influenzare l'investimento lavorativo e l'utilizzo di strategie proattive migliorando sia l'adattamento che la soddisfazione lavorativa dei docenti e il generale clima in classe (Mason et al., 2014; Mauno, Ruokolainen, Kinnunen, \& De Bloom, 2016).

Infine, tra gli aspetti disposizionali che possono incidere sul benessere lavorativo degli insegnanti si può annoverare l'affettività disposizionale positiva che riflette il massimo grado con cui un individuo riporta di sentirsi entusiasta, attivo e vigile (Watson et al., 1988). La presenza di un'affettività disposizionale positiva, come forma di regolazione emotiva, consentirebbe alle insegnanti di affrontare lo stress (Tugade \& Friedrickson, 2004). Come mostrano alcuni studi, le persone che riportano un'elevata affettività positiva risultano essere più energici, di buon umore $\mathrm{e}$ tendono a ricercare con più facilità supporto sociale (Watson et al., 1999). Inoltre, una meta-analisi del 2003 (Thoresen, Kaplan, Barsky, Warren, \& DeChermont, 2003) ha messo in evidenza come un'elevata affettività positiva sia correlata positivamente con l'efficacia professionale e negativamente con esaurimento emotivo e cinismo (Kahn, Schneider, Jenkins-Henkelman, \& Moyle, 2006).

\section{Strategie proattive nel contesto educativo e scolastico}

In letteratura con il termine "strategie di coping" nel contesto scolastico ci si riferisce agli sforzi cognitivi e comportamentali messi in atto dagli insegnanti per ridurre, tollerare 0 gestire lo stress correlato al lavoro (Carmona, Buunk, Peiro, Rodríguez, \& Bravo, 2006; Lazarus, 1993; Parker, Martin, Colmar, \& Liem, 2012; Sharplin, 0'Neill, \& Chapman, 2011). Nello specifico vengono definite autoregolative le strategie generate dall'insegnante stesso al fine di affrontare situazioni stressanti a lavoro (Boekaerts, Pintrich, \& Zeidner, 2005; Zimmerman, 2002), mentre vengono definite coregolative, le strategie con cui l'insegnante affronta lo stress ricorrendo all'utilizzo di risorse sociali (Järvelä, Volet, \& Järvenoja, 2010). Queste strategie avrebbero lo scopo di aiutare gli individui ad orientare i propri comportamenti, al fine di raggiungere obiettivi prefissati, e ridurrebbero il rischio di burnout (Butler, 2007; Devos, Dupriez, \& Paquay, 2012; Xanthopoulou, Bakker, Demerouti, \& Schaufeli, 2009). Inoltre, le persone che utilizzano strategie attive piuttosto che strategie di evitamento, risulterebbero più efficaci nell'affrontare le sfide e le richieste provenienti dal proprio 
contesto lavorativo e sarebbero anche più soddisfatti e più coinvolti nel loro lavoro (Salmela-Aro, Tolvanen, \& Nurmi, 2011). Anche le emozioni positive promuoverebbero un uso più flessibile di queste strategie (Fredrickson, 2001) così come la tendenza a trovare sempre nuove e originali idee per far fronte a situazioni problematiche (Linnenbrink-Garcia, Kempler, Rogat, \& Koskey, 2011; Lyubomirsky, King, \& Diener, 2005). Gli studi condotti nel contesto scolastico hanno evidenziato come insegnanti che sperimentano una spinta positiva nei confronti del loro lavoro e uno stato di empowerment, tenderebbero ad utilizzare strategie attive, riflessive ed olistiche per risolvere i problemi (Soini, Pyhältö, e Pietarinen, 2010). Inoltre, considerata la natura sociale e interazionale del contesto scolastico, gli insegnanti potrebbero con più facilità co-regolare i loro comportamenti. Un clima sociale positivo, caratterizzato da elevati livelli di supporto sociale, sarebbe correlato a maggiori livelli di soddisfazione e motivazione (Alhija \& Fresko, 2010; Skaalvik \& Skaalvik, 2011) e a minori livelli di burnout (e.g. Hakanen, Bakker, \& Schaufeli, 2006). Infatti, gli insegnanti supportati dai propri colleghi sarebbero meno intenzionati ad abbandonare il proprio lavoro rispetto ai colleghi che, invece, lavorano in un contesto caratterizzato da basso supporto sociale (Pomaki, DeLongis, Frey, Short, \& Woehrle, 2010). Nel contesto scolastico, la percezione di autoefficacia del singolo appare profondamente legata al funzionamento del gruppo. La percezione di autoefficacia collettiva rappresenta l'insieme di credenze da parte dei docenti rispetto alle abilità dei colleghi di predisporre schemi di azione efficaci nel raggiungimento di obiettivi specifici. Solo pochi contributi hanno esaminato tale costrutto, ipotizzando che la percezione di autoefficacia collettiva rappresenti un elemento determinante per l'esperienza lavorativa del singolo (Goddard, Hoy, \& Hoy, 2004).

Alcuni studi hanno evidenziato il ruolo cruciale giocato dalle strategie proattive nel ridurre lo stress delle insegnanti e nel promuovere un miglior contesto lavorativo (Bermejo-Toro, Prieto-Ursúa, \& Hernández, 2016; Pietarinen, Pyhältö, Soini, \& Salmela-Aro, 2013; Tikkanen, Pyhältö, Pietarinen \& Soini, 2017). L'importanza di tenere in considerazione queste strategie risiede nel fatto che il loro uso risulta strettamente legato alle interazioni sociali presenti nella scuola in cui gli insegnanti lavorano e, di conseguenza, esse potrebbero essere oggetto di interventi di promozione e sostegno (Pietarinen, Pyhältö, Soini, \& Salmela-Aro, 2013). Le strategie proattive che vengono adottate dagli insegnanti, quindi, potrebbero non solo ridurre il rischio di burnout ma anche contribuire allo sviluppo di un contesto lavorativo positivo ed incrementare il coinvolgimento lavorativo.

\section{Coinvolgimento Lavorativo e l'Adattamento insegnante-ambiente di lavoro}

Il coinvolgimento lavorativo (work engagement) è stato oggetto di numerosi approfondimenti nel corso degli ultimi decenni ed è stato dimostrato essere un fattore predittivo della performance e del livello di benessere lavorativo sia dei singoli che delle organizzazioni (Kahn \& Scott, 1997; Bakker et al, 2008). Secondo Schaufeli e Bakker (2004), il work engagement sarebbe definibile come un senso di coinvolgimento nel proprio lavoro associato a buona produttività e a benessere psicofisico, e sarebbe la risultante di tre dimensioni, una fisica (vigore), una emotiva (dedizione) e una cognitiva (coinvolgimento) (Schaufeli, Bakker, \& Salanova, 2006; Salanova \& Schaufeli, 2009). Gli studi condotti fino ad oggi hanno confermato la relazione fra benessere soggettivo, inteso come risorsa personale, e l'engagement a lavoro (Bakker \& Oerlemans, 2016; Field \& Buitendach, 2011; Huynh, Xanthopoulou, \& Winefield, 2014; Rodríguez-Muñoz, Sanz-Vergel, Demerouti, \& Bakker, 2014) ma sono ancora pochi quelli che indagano questa relazione all'interno contesto scolastico (Chan, 2009; Pillay, Goddard, \& Wilss, 2005; Authors, 2017). Più precisamente, il benessere soggettivo è sempre stato considerato come una conseguenza del work engagement e, nonostante diversi studi si siano concentrati su risorse personali come resilienza e ottimismo in termini di antecedenti del work 
engagement, sembrerebbero non esserci studi che valutino gli effetti del benessere soggettivo degli insegnanti sul loro work engagement (Halbesleben, 2010; Xanthopoulou, Bakker, Demerouti, \& Schaufeli, 2007). In generale l'ingaggio a lavoro nel contesto scolastico è emerso essere predittivo della performance, di una bassa intenzione ad abbandonare l'insegnamento, e influenzerebbe la relazione positiva con gli studenti e i risultati accademici degli stessi (Bakker, Schaufeli, Leiter \& Taris, 2008; Bakker, Van Emmerik \& Euwema, 2006; Duckworth, Quinn, \& Seligman, 2009; Salanova, Schaufeli, Xanthopoulou, \& Bakker, 2010).

Se prendiamo in considerazione altre risorse personali che possono promuovere il work engagement, la compassione a lavoro è emersa essere un forte fattore predittivo (Mason et al. 2014; Mauno, Ruokolainen, Kinnunen, \& De Bloom, 2016). Nello specifico per quanto riguarda gli insegnanti, il coinvolgimento a lavoro è risultato collegato al benessere soggettivo e alla compassione fra le insegnanti e, più nello specifico, la compassione sembra essere positivamente legata al vigore, alla dedizione e alla soddisfazione degli insegnanti e negativamente al burnout, risultando quindi essere uno strumento importante per affrontare lo stress legato all'esercizio della professione (Eldor \& Shoshani, 2016).

Inoltre, un clima lavorativo positivo (Hoy \& Spero, 2005), in cui gli insegnanti percepiscono di ricevere, da colleghi e superiori, feedback costruttivi e riconoscimento professionale (Kokkinos, 2007; Peeters \& Rutte, 2005; Stoeber \& Rennert, 2008), potrebbe giocare un ruolo chiave nel promuovere soddisfazione e coinvolgimento lavorativo che, a loro volta, migliorerebbero le performance dei lavoratori (Klusmann, Kunter, Trautwein, Ludtke, \& Baumert, 2008; Xanthopoulou, Bakker, Demerouti, \& Schaufeli, 2009).

\section{METODO}

L' obiettivo del presente studio di carattere quali-quantitativo è stato duplice:1) valutare il ruolo predittivo, sul coinvolgimento lavorativo degli operatori dei servizi educativi e scolastici 0/6 anni, di variabili disposizionali (benessere soggettivo, compassione, affettività disposizionale positiva) e di natura socio-contestuale (strategie proattive auto ed etero regolative, clima costruttivo); 2) esplorare sul piano qualitativo le dimensioni di promozione del benessere nei servizi.

\section{Partecipanti e Procedura}

Sono stati coinvolti 189 educatori ed insegnanti della scuola dell'infanzia ( $89 \%$ donne) reclutati presso i servizi educativi e scolastici di Roma Capitale. L'età dei partecipanti varia da 27 a 63 anni (età media $\mathrm{M}=36.03 \mathrm{DS}=10.15$ ). Rispetto allo stato coniugale, il $56.5 \%$ risulta sposato, il $21.6 \%$ single, il $18.4 \%$ separato/divorziato, e 1 persona riferisce di essere vedova.

I partecipanti hanno preso parte allo studio, su base volontaria, compilando i questionari, somministrati collettivamente, in forma anonima. Le autrici hanno organizzato riunioni plenarie nelle scuole al fine di informare gli insegnanti sulle finalità della ricerca e sulle modalità di compilazione del protocollo di ricerca. La ricerca è stata condotta secondo i principi etici dell'APA (American Psychological Association, 2017).

\section{Strumenti}

Subjective Happiness Scale (SHS; Lyubomirsky \& Lepper, 1999). La SHS è una scala composta da 4 item che valuta la felicità soggettiva. Nello specifico, il primo e il secondo item chiedono ai soggetti di valutare su una scala Likert a 7 punti quanto generalmente si sentano felici $(1=$ una persona non molto felice, 7 = una persona molto felice) e quanto si considerino felici rispetto ai 
propri pari ( 1 = una persona non molto felice, 7 = una persona molto felice); il terzo e il quarto item, invece, chiedono ai soggetti di indicare quanto le descrizioni di una persona "molto felice" o "non molto felice" li rappresenti $(1=$ per nulla, $7=$ molto). Punteggi elevati a questa scala sono indice di una maggiore felicità soggettiva. Gli studi precedenti hanno riportato per questa scala un coefficiente $\alpha$ di Cronbach che varia da .79 a .94 (Lyubomirsky \& Lepper, 1999).

Santa Clara Brief Compassion Scale (SCBCS; Hwang et al., 2008). La SCBCS è una scala a 5 item che valuta la compassione e la sua connessione ai comportamenti pro-sociali. Gli item che la compongono fanno riferimento a due costrutti della compassione: la connessione emotiva con persone che vivono momenti difficili e l'agire per aiutarle. Ė una versione ridotta della Sprecher and Fehr's (2005) "Compassionate Love Scale" (la correlazione fra le due scale è di $r=0.95$ ) che fa riferimento esclusivamente a persone con cui non si hanno relazioni personali profonde (es. estranei). Tutti gli item sono misurati su scala likert a 7 punti $(1=$ molto in disaccordo, $7=$ molto d'accordo) e punteggi elevati sono indicativi di grande compassione.

Utrecht Work Engagement Scale (UWES-17; Schaufeli \& Bakker, 2004). La variabile Work Engagement è stata valutata attraverso la versione italiana della UWES-17 (Balducci, Fraccaroli, and Schaufeli, 2010). La scala è composta da 17 item misurati su scala Likert a 6 passi (1= Quasi mai, $6=$ Sempre) e comprende tre sottoscale: vigore (6 item), dedizione (5 item), e coinvolgimento (6 item). II coefficiente $\alpha$ di Cronbach risulta essere pari a .96.

The Proactive Strategy scale (Salmela-Aro,2009). La scala è composta da 7 item che misurano due fattori che caratterizzano le strategie proattive: a) auto-regolazione (4 item) e b) co-regolazione (3 item). Tutti gli item sono misurati su scala Likert a 7 punti che comprende valori da 1 (molto in disaccordo) a 7 (molto d'accordo).

The Teacher-working environment fit scale (Pyhältö, Pietrarinen, \& Salmela-Aro, 2011) è una scala composta da 6 item che misura due dimensioni principali dell'adattamento insegnanteambiente di lavoro: a) riconoscimento professionale ricevuto da parte dei colleghi ( 3 item) e b) clima lavorativo positivo e costruttivo (3 item). La prima dimensione misura il riconoscimento professionale percepito dall'insegnante in termini di accettazione come membro di una comunità professionale. La seconda dimensione misura la capacità degli insegnanti di contribuire ad un adattamento ottimale all'interno del gruppo professionale, così come percepita dagli insegnanti stessi (i.e. approccio centrato sulla persona riguardo l'adattamento percepito). Tutti gli item sono valutati per mezzo di una scala Likert a 7 punti che comprende valori da 1 (completamente in disaccordo) a 7 (completamente d'accordo).

The Positive and Negative Affect Schedule (PANAS; Watson et al. 1988) misura due dimensioni dell'affettività disposizionale individuale, una positiva e una negativa. Ai partecipanti è stato chiesto di valutare "quanto si sentono cosi generalmente" in relazione a 20 emozioni (come "ostile" e "entusiasta") utilizzando una scala Likert a 5 punti ( $1=$ "molto poco/per niente", $5=$ "moltissimo"). I punteggi dei 10 item appartenenti alla sottoscala positiva e quelli dei 10 appartenenti alla sottoscala negativa vengono sommati separatamente per ottenere i punteggi globali delle due sottoscale. Ciascuna dimensione ottiene un punteggio complessivo che varia da 10 a 50 e punteggi elevati sono indicativi della presenza di maggiori affetti positivi/negativi.

La Teacher self-efficacy degli insegnanti è stata misurata con una scala creata ad hoc, composta da 5 item seguendo le raccomandazioni di Bandura $(1996,2006)$. L'autoefficacia dell'insegnante è stata misurata tramite cinque componenti fondamentali di tale costrutto: gestione degli studenti considerati "difficili", uso delle nuove tecnologie, fronteggiamento delle sfide educative, collaborazione con i colleghi, correlazione dei target e degli obiettivi didattici. Tutti gli item sono stati 
graduati su una scala Likert a 5 passi: quasi mai (1), raramente (2), qualche volta (3), spesso (4), sempre (5).

Intervista semi-strutturata costruita ad hoc che valuta gli aspetti di benessere individuabili nell'esercizio della propria professione.

\section{Analisi e Risultati}

Al fine di indagare le relazioni esistenti fra le variabili oggetto di studio, sono state effettuate analisi di correlazione bivariata (Tabella 1). A partire dagli esiti statisticamente più rilevanti si evidenzia come all'aumentare del benessere soggettivo percepito dagli insegnanti, aumentino anche i livelli di emozionalità positiva disposizionale $(r=.20, p<0.01)$, di strategie proattive utilizzate $(r=$ $.23, p<0.01)$ di work engagement $(r=.37, p<0.01)$. All'aumentare della compassione si registra un aumento di livelli di work engagement $(r=.24, p<0.01)$. All'aumentare dei livelli di affettività positiva disposizionale aumentano l'efficacia collettiva percepita $(r=.23, p<0.01)$, i livelli di work engagement $(r=.32, p<0.01)$ e le strategie proattive utilizzate $(r=.22, p<0.01)$. Infine, un maggiore utilizzo di strategie proattive utilizzate dagli insegnanti si associa con un più alto livello di benessere soggettivo percepito $(r=.23, p<0.01)$, di affettività positiva disposizionale $(r=.22, p<$ $0.01)$, di efficacia collettiva percepita $(r=.37, p<0.01)$, di livelli di work engagement $(r=.40, p<$ $0.01)$, di riconoscimento professionale percepito $(r=.47, p<0.01)$ e la capacità degli insegnanti di contribuire ad un clima costruttivo del gruppo professionale $(r=.48, p<0.01)$.

Alla luce delle correlazioni emerse, è stata successivamente effettuata un'analisi di regressione multipla lineare gerarchica a 3 step (Tabella 2) nella quale sono state inserite come variabile dipendente i livelli di work engagement percepiti dagli insegnanti e come predittori variabili sociodemografiche (età e stato civile) al primo step, variabili disposizionali quali benessere soggettivo, compassione e affettività positiva al secondo, e capacità degli insegnanti di contribuire ad un adattamento ottimale all'interno del gruppo professionale, efficacia collettiva e strategie proattive, al terzo. II modello finale spiega il $32 \%$ della variabilità del fenomeno studiato $\left(\mathrm{R}^{2}=.32, \mathrm{~F}_{(8,306)}=\right.$ 18.06, $\mathrm{p}<0.001)$. I risultati evidenziano come a parità di aspetti di benessere $\left(\beta=.257, \mathrm{t}_{(313)}=\right.$ 5.208, $\mathrm{p}=.000)$, compassione $\left(\beta=.183, \mathrm{t}_{(313)}=3.832, \mathrm{p}=.000\right)$ e di affettività disposizionale positiva $\left(\beta=.183, t_{(313)}=3.680, p=.000\right)$, le strategie proattive $\left(\beta=.260, t_{(313)}=4.627, p=.000\right)$ aumentino la probabilità dei docenti di avere maggior engagement a scuola.

E' stato inoltre chiesto ai partecipanti di indicare quali fossero le dimensioni in grado di promuovere il loro benessere nel contesto educativo e scolastico.

Attraverso l'analisi qualitativa dei contenuti delle risposte effettuata da due giudici indipendenti è stato possibile individuare le seguenti macrocategorie contenutistiche: (1) dimensione relazionale con alunni e famiglie; (2) dimensione relazionale con colleghi e coordinatori; (3) risorse personali; (4) aspetti organizzativi relativi al contesto educativo e scolastico; (5) esiti positivi del percorso educativo degli alunni; (6) riconoscimento dell'attività lavorativa da parte di famiglie, colleghi e superiori. E' stata condotta un'analisi delle frequenze dei contenuti delle risposte fornite dai partecipanti rispetto alle macro aree contenutistiche individuate (Grafico 1), dalla quale emerge che il $41 \%$ dei partecipanti riconosce la collaborazione con i colleghi e la presenza di dirigenti scolastici competenti come un aspetto centrale nel proprio benessere lavorativo; il $20 \%$ riporta come elemento saliente del benessere a scuola la presenza di un ambiente ben organizzato, dotato di spazi e strumenti appropriati, con una definita divisione di ruoli e competenze; il 18\% del campione ha riportato come aspetto di benessere nel proprio contesto lavorativo il rapporto positivo con gli alunni 
e con le famiglie; il 14\% individua alcune dimensioni personali individuali (motivazione, creatività e empatia) come risorse importanti per una ottimale gestione del proprio lavoro; II 5\% riporta come sentirsi valorizzati, apprezzati e ricevere attenzioni per il lavoro svolto risulti essere un fattore importante per il benessere nel contesto lavorativo ed infine solo il $2 \%$ ha fatto riferimento agli esiti positivi dei percorsi educativi ottenuti dagli alunni, come dimensione in grado di promuovere benessere nel contesto educativo e scolastico.

\begin{tabular}{|c|c|c|c|c|c|c|c|c|c|c|}
\hline & 1 & 2 & 3 & 4 & 5 & 6 & 7 & 8 & 9 & 10 \\
\hline 1. Età & 1 & & & & & & & & & \\
\hline 2. Stato Civile &, $43^{* *}$ & 1 & & & & & & & & \\
\hline 3. Benessere soggettivo &, 06 &, $14^{*}$ & 1 & & & & & & & \\
\hline 4. Compassione &,- 02 &,- 05 &, 05 & 1 & & & & & & \\
\hline 5. Affettività positiva &, 03 &, 06 & $20^{* * *}$ &, 05 & 1 & & & & & \\
\hline 6. Efficacia collettiva &, 04 &, 01 &, 08 &, 09 &, $23^{* *}$ & 1 & & & & \\
\hline 7. Work engagement &, 02 &, 03 & $37^{* * *}$ &, $24^{* *}$ &, $32^{* *}$ &, $25^{* *}$ & 1 & & & \\
\hline $\begin{array}{l}\text { 8. Riconoscimento } \\
\text { professionale }\end{array}$ & ,06 &, $12^{*}$ &, $18^{* *}$ & ,07 &, $14^{*}$ &, $38^{* *}$ &, $30^{* *}$ & 1 & & \\
\hline 9. Clima costruttivo &,- 00 &,- 01 &, $13^{*}$ &, $15^{* *}$ &, $13^{*}$ & $65^{* *}$ &, $27^{* *}$ &, $58^{* * *}$ & 1 & \\
\hline 10. Strategie proattive &, 08 &, $14^{*}$ & $23^{* *}$ &, 09 &, $22^{* *}$ &, $37^{* *}$ &, $40^{* *}$ &, $47^{* *}$ &, $48^{* *}$ & 1 \\
\hline
\end{tabular}

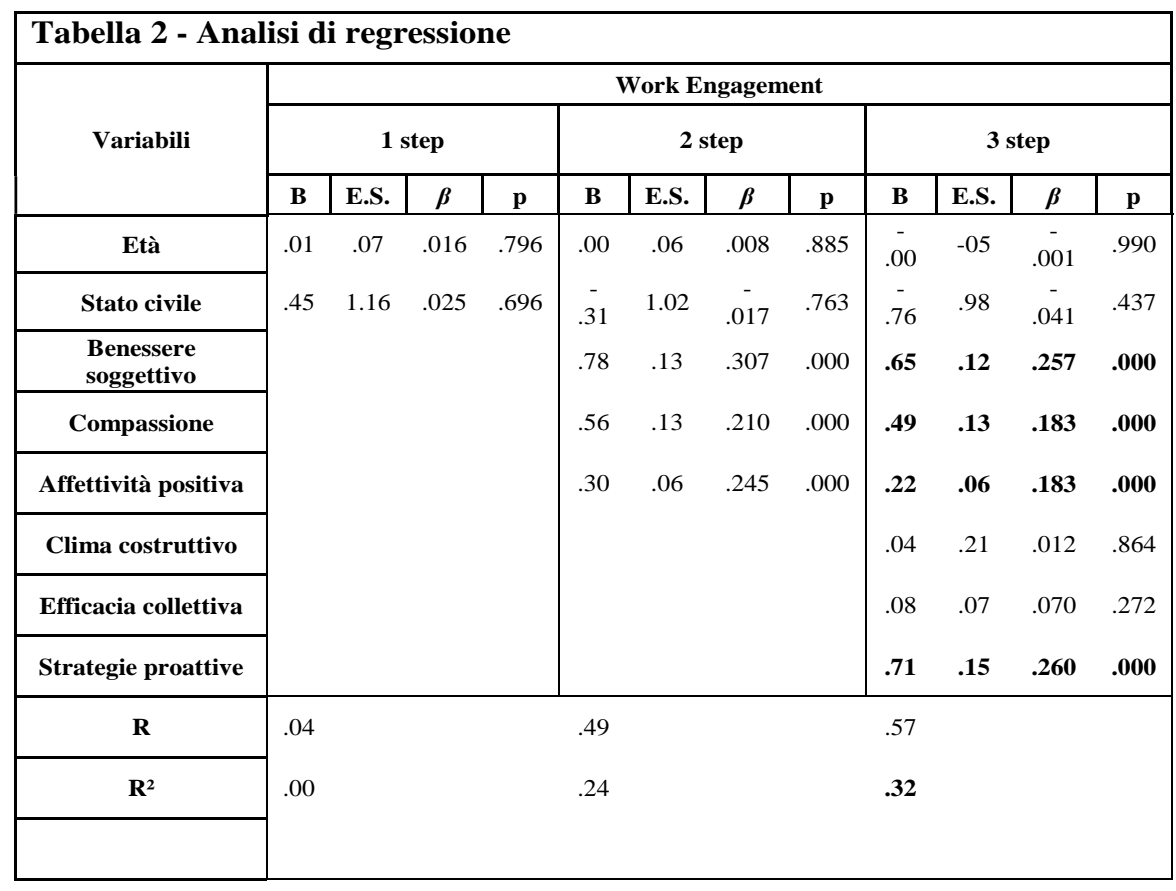






\section{Discussione}

II presente contributo si inserisce nel filone di studi che, a partire da una prospettiva empirica di Psicologia Positiva, si propone di mettere in luce i fattori in grado di promuovere il coinvolgimento lavorativo dei docenti all'interno dei servizi $0 / 6$ anni.

L' obiettivo del presente studio di carattere quali-quantitativo è stato duplice: 1) valutare il ruolo predittivo, sul coinvolgimento lavorativo degli operatori dei servizi educativi e scolastici 0/6 anni, di variabili disposizionali (benessere soggettivo, compassione, affettività disposizionale positiva) e di natura socio-contestuale (strategie proattive auto ed etero regolative, clima costruttivo, senso di autoefficacia collettiva); 2) esplorare sul piano qualitativo le dimensioni di promozione del benessere nei servizi.

I principali esiti del modello di regressione proposto mostrano come il coinvolgimento lavorativo in ambito educativo e scolastico sia riconducibile, oltre a diversi fattori disposizionali individuali (quali benessere soggettivo, compassione e affettività positiva), anche all'utilizzo da parte dei docenti di strategie proattive auto ed etero-regolative.

Tali esiti sono coerenti con la letteratura presente sul tema. In accordo con Bakker e colleghi (2014), il benessere soggettivo promuove il coinvolgimento lavorativo, ponendo gli insegnanti nella condizione di utilizzare al meglio le proprie risorse, sia sul piano individuale che relazionale (Bakker \& Oerlemans, 2016; Field \& Buitendach, 2011; Huynh, Xanthopoulou, \& Winefield, 2014), con effetti positivi sulla qualità dell'insegnamento (Bakker et al., 2014). Alcuni contributi hanno infatti descritto come il benessere disposizionale sul luogo di lavoro migliorerebbe la performance lavorativa, il supporto sociale ricevuto e il successo lavorativo (Boehm \& Lyubomirsky, 2008; Burger \& Caldwell, 2000).

In linea con la "Broaden-and-build Theory of Positive Emotions" di Fredrickson (2001), inoltre, fare esperienza sul luogo di lavoro di stati affettivi positivi, generati da una condizione di benessere individuale e dalla condivisione di condotte compassionevoli da parte e nei riguardi dei colleghi, stimolerebbe le risorse personali, cognitive e comportamentali dei singoli attori che intervengono 
nel contesto lavorativo, favorendo maggiore impegno e dedizione al proprio lavoro (Fredrickson, 2001; 2013; Cropanzano \& Wright, 2001; Fredrickson \& Losada, 2005; Kok et al., 2013).

In linea con i nostri risultati, altri contributi hanno evidenziato la relazione positiva esistente fra condotte compassionevoli e coinvolgimento lavorativo (Tremblay and Messervey 2011; Eldor, 2018; Mason et al., 2014). In particolare, Eldor (2016), descrive come in ambito educativo e scolastico la compassione influisca in maniera significativa sugli outcome lavorativi a lungo termine, specialmente quando espressa da pari grado o colleghi di grado superiore. Ricevere compassione da parte dei colleghi sembra porre gli insegnanti nella condizione di sperimentare maggiore senso di autoefficacia personale, facendo esperienza del contesto lavorativo scolastico come orientato al prendersi cura dell'altro, tanto degli allievi quanto dei colleghi. In accordo con Underwood (2009) sperimentare compassione comporta, infatti, una valutazione dell'altro orientata alla comprensione dei suoi bisogni e delle sue emozioni, attraverso una disposizione personale volta all'accoglienza e alla reciprocità. Secondo quanto descritto in letteratura, sperimentare e mettere in atto condotte compassionevoli produce un maggior riconoscimento del proprio ruolo d'insegnante, supportando $\mathrm{i}$ docenti nella gestione di aspetti critici del lavoro, quali ad esempio i comportamenti problematici degli studenti (Dutton, Worline, Frost, \& Lilius, 2006; Hargreaves, 2000; Lilius et al., 2008).

Allo stesso modo, l'affettività positiva si configura come una modalità disposizionale di regolazione affettiva che riflette il coinvolgimento positivo di ognuno nell'ambiente in cui si trova (Diener et al, 2017). Nel campione considerato, tale dimensione partecipa nel determinare migliori livelli di coinvolgimento percepito da parte degli insegnanti. Alti livelli di affettività positiva sono riportati in letteratura come correlati a uno stato di entusiasmo, allerta, energia, gioia, interesse e determinazione. L'affettività positiva, inducendo un'esperienza emotiva positiva e piacevole, infatti, si riflette in un generale senso di benessere, competenza e coinvolgimento all'interno di relazioni interpersonali percepite come efficaci (Watson \& Tellegen, 1985; Watson, Clark, \& Carey, 1988). Con riferimento all'ambiente scolastico, come riportato da Eldor (2016), I'affettività positiva è descritta come un fattore di mediazione nella gestione di elevate fonti di stress, sostenendo gli insegnanti nella gestione di condizioni particolarmente stressanti, mantenendo vigore emotivo, impegno organizzativo e soddisfazione lavorativa.

In accordo con la letteratura, fattori soggettivi disposizionali concorrono nel determinare il modo in cui le risorse lavorative sono percepite (Bakker, Demerouti, \& Sanz-Vergel, 2014); queste ultime appaiono di primaria importanza nel determinare la qualità dell'esperienza lavorativa.

Gli esiti delle analisi svolte mostrano come I'utilizzo di strategie proattive a orientamento relazionale aumenti la probabilità per gli educatori e gli insegnanti della scuola dell'infanzia di sperimentare un maggior coinvolgimento lavorativo. L'utilizzo di strategie di coping proattive, in particolare volte alla co-regolazione, si declina nell'identificazione e nell'uso attivo di tutte le risorse sociali disponibili, costituendo un valido aiuto nella gestione delle richieste lavorative (Järvelä, Volet, \& Järvenoja, 2010).

I nostri risultati, in linea con gli esiti descritti in letteratura, sembrano suggerire l'importanza delle dinamiche relazionali che occorrono nel contesto scolastico. Adottare strategie proattive permette agli insegnanti di regolare il proprio comportamento, in relazione e in accordo con le condizioni dell'ambiente di lavoro in cui questi sono inseriti, favorendone l'adattamento. Pietarinen, Pyhältö, Soini, e Salmela-Aro (2013) hanno messo in evidenza la relazione fra l'uso di strategie proattive da parte di un gruppo di insegnanti, il livello di disagio avvertito sul luogo di lavoro e la percezione da parte degli stessi del proprio adattamento, sottolineando come interventi specifici possano promuovere attivamente il loro impiego. Bakker, Tims, e Derks (2012) descrivono come un 
assetto personologico volto alla proattività sia legato al coinvolgimento lavorativo, manifestandosi in un'attiva ricerca di confronto con i colleghi in merito al lavoro svolto, nella creazione di opportunità di sviluppo, nel proporre obiettivi e pianificare nuove progettualità. L'utilizzo di strategie sociali sostiene i soggetti nella ricerca di nuove soluzioni ai problemi, promuovendo un uso flessibile delle strategie di coping disponibili (Linnenbrink-Garcia, Kempler, Rogat, \& Koskey, 2011) con effetti sul proprio benessere lavorativo. La soddisfazione lavorativa degli insegnanti e la loro motivazione sarebbero legate alla presenza di un clima percepito come presente e supportivo; tali aspetti inducono una riduzione del rischio di abbandono del posto di lavoro, anche quando le richieste lavorative risultano particolarmente intense (Pomaki, DeLongis, Frey, Short, \& Woehrle, 2010).

L'individuazione dei fattori capaci di promuovere il coinvolgimento lavorativo degli insegnanti permette di promuovere il benessere lavorativo dei docenti, prevenendo il rischio di disagio, del burnout e del turnover.

L'importanza degli aspetti relazionali ed emotivi è stata confermata nel presente studio anche rispetto a quanto riferito dai docenti nelle domande aperte che sono state poste loro sugli aspetti di promozione del benessere nel contesto educativo e scolastico. L'importanza della trama relazionale si declina non solo nel rapporto con gli studenti, ma in particolar modo nella collaborazione con le famiglie, i colleghi e i superiori (Albanese 2009; Fiorilli et al., 2015; Cummings, 2016).

La collaborazione con i colleghi, concordando obiettivi di lavoro comuni, all'interno di un gruppo disponibile al dialogo, supportivo e collaborativo, viene riconosciuta da una larga percentuale del nostro campione (41\%) come un aspetto centrale del proprio benessere lavorativo; a ciò si aggiunge la presenza di dirigenti scolastici competenti, vigili e attenti alle dinamiche che occorrono nell'ambiente di lavoro. Tali elementi trovano ampio riscontro in letteratura (Hakanen, 2006; Schreyer e Krause, 2016; Løvgren, 2016; Hur et al., 2016).

Sentirsi valorizzati, apprezzati e ricevere attenzioni per il lavoro svolto non solo dai colleghi, dai supervisori e coordinatori, ma anche da parte dei piccoli alunni e delle loro famiglie, risulta essere un fattore importante per il lavoro degli operatori dei servizi educativi e scolastici. In linea con quanto da noi osservato, Bakker, Hakanen, Demerouti e Xanthopoulou (2007) hanno identificato il supporto ricevuto dai propri superiori, l'apprezzamento per il lavoro svolto e, più in generale il clima organizzativo, come importanti risorse, utili per gli insegnanti nel fronteggiare le criticità che caratterizzano il complesso rapporto con i propri studenti. Per il 18\% del campione, il rapporto con gli alunni e con le famiglie rappresenta un aspetto centrale nell'esercizio della professione. La presenza di un clima positivo in classe concorre nel determinare il benessere sperimentato dai docenti, in accordo con quanto descritto in letteratura (Jennings e Greenberg, 2009; Chang, 2009); a questa dimensione si affianca, come aspetto influente sulla qualità dell'esperienza dell'insegnamento, la collaborazione con le famiglie e la partecipazione attiva al processo educativo.

Anche se solo per una percentuale limitata del nostro campione (2\%), gli esiti positivi del percorso educativo ottenuti dai piccoli alunni e gli obiettivi formativi da loro conseguiti rappresentano un elemento di benessere per gli insegnanti nell'esercizio della propria professione.

La presenza di un ambiente ben organizzato, dotato di spazi adeguati, attrezzature e strumenti appropriati a sostegno della didattica, con una definita divisione di ruoli e competenze è riportato nel $20 \%$ dei casi come elemento saliente del benessere percepito a scuola. Tale assunto trova riscontro in letteratura (Schreyer e Krause 2016). La necessità di un'attenta pianificazione delle attività didattiche, condivisa fra i colleghi, risulta essere un elemento largamente condiviso dai docenti: Blöchliger e Bauer (2015) descrivono, infatti, come in ambito scolastico la gestione del carico di lavoro e le condizioni in cui questo viene svolto, possono influenzare la percezione delle 
risorse che gli insegnanti hanno a loro disposizione, con effetti sull'esercizio stesso della professione.

In questo panorama, il $14 \%$ del nostro campione individua in dimensioni personali individuali quali la motivazione, la creatività e l'empatia, risorse importanti per un'ottimale gestione del proprio lavoro. Alcuni studi recenti hanno considerato il ruolo delle risorse personali sul disagio e sul coinvolgimento lavorativo nell'ambito della professione dell'insegnamento, individuando le relazioni fra questi intercorrenti (Bakker et al., 2007; Xanthopoulou et al., 2007; Bermejo-Toro, Prieto-Ursùa, \& Hernandez, 2015).

I nostri risultati sottolineano l'importanza di considerare l'area delle risorse personali e del contesto di lavoro nella prospettiva di incrementare il benessere lavorativo e prevenire il disagio attraverso interventi specifici. Prospettive future possono includere un'approfondita analisi qualitativa dei vissuti degli insegnanti, superando il limite dell'utilizzo di strumenti self-report. Sarebbe inoltre interessante approfondire le relazioni fra i costrutti all'interno di una prospettiva longitudinale, considerando non solo le relazioni fra le dimensioni prese in esame, ma i rapporti di causalità esistenti fra le stesse. Ci sembra importante sottolineare come gli esiti dello studio offrano un'utile panoramica rispetto ai principali fattori protettivi del coinvolgimento lavorativo, fornendo utili spunti per i programmi volti alla promozione del benessere degli educatori e degli insegnanti della scuola dell'infanzia. Ci sembra utile, a questo proposito, ribadire come la formazione degli insegnanti possa rappresentare sia uno spazio essenziale per fornire loro strumenti che li aiutino a prendere consapevolezza delle proprie risorse, sia un momento di condivisione in cui aprirsi, insieme ai propri colleghi, a momenti di reale collegialità. In particolare riteniamo fondamentale, in una prospettiva di Psicologia Positiva, pensare ad azioni formative che partano dall'analisi dei punti di forza del contesto e dei soggetti, dalla circoscrizione delle zone problematiche e dall'attivazione di una rete il più ampia possibile, al fine di favorire il superamento delle difficoltà.

\section{BIBLIOGRAFIA}

Albanese 0., Pepe A., Fiorilli C., Gabola P. (2014), "Emozioni, supporto sociale ed esaurimento emotivo negli insegnanti: una relazione complessa", Psicologia e Scuola, 1, 15-22.

Alhija, F. N. A., \& Fresko, B. (2010). Socialization of new teachers: Does induction matter?. Teaching and teacher education, 26(8), 1592-1597.

American Psychological Association. (2017). Ethical principles of psychologists and code of conduct. Retrieved from: https://www.apa.org/ethics/code/ethics-code-2017.pdf

Bakker, A. B., Demerouti, E., \& Sanz-Vergel, A. I. (2014). Burnout and work engagement: The JD-R approach. Annu. Rev. Organ. Psychol. Organ. Behav., 1(1), 389-411.

Bakker, A. B., Emmerik, H. V., \& Euwema, M. C. (2006). Crossover of burnout and engagement in work teams. Work and occupations, 33(4), 464-489.

Bakker, A. B., Hakanen, J. J., Demerouti, E., \& Xanthopoulou, D. (2007). Job resources boost work engagement particularly when job demands are high. Journal of Educational Psychology, 99(2), 274-284.

Bakker, A. B., \& Oerlemans, W. G. (2016). Momentary work happiness as a function of enduring burnout and work engagement. The Journal of psychology, 150(6), 755-778.

Bakker, A. B., Schaufeli, W. B., Leiter, M. P., \& Taris, T. W. (2008). Work engagement: An emerging concept in occupational health psychology. Work \& Stress, 22(3), 187-200. 
Bakker, A. B., Tims, M., \& Derks, D. (2012). Proactive personality and job performance: The role of job crafting and work engagement. Human relations, 65(10), 1359-1378.

Balducci, C., Fraccaroli, F., \& Schaufeli, W. B. (2010). Psychometric properties of the Italian version of the Utrecht Work Engagement Scale (UWES-9). European Journal of Psychological Assessment, 26(2), 143-149.

Bandura, A. (1996). Social cognitive theory of human development. International encyclopedia of education, 2, 5513-5518.

Bandura, A. (2006). Guide for constructing self-efficacy scales. Self-efficacy beliefs of adolescents, 5(1), 307-337.

Baumeister, R. F., \& Leary, M. R. (1995). The need to belong: Desire for interpersonal. The Use of Oral Narrative in North American Families, 159.

Bermejo-Toro, L., Prieto-Ursúa, M., \& Hernández, V. (2016). Towards a model of teacher well-being: Personal and job resources involved in teacher burnout and engagement. Educational Psychology, 36(3), 481-501.

Bloechliger, 0. R., \& Bauer, G. F. (2016). Demands and job resources in the child care workforce: Swiss lead teacher and assistant teacher assessments. Early Education and Development, 27(7), 1040-1059.

Boekaerts, M., Pintrich, P. R., \& Zeidner, M. (2005). Handbook of Self. Regulation, 451-502.

Boehm, J. K., \& Lyubomirsky, S. (2008). Does happiness promote career success?. Journal of career assessment, 16(1), 101-116.

Borman, W. C., Penner, L. A., Allen, T. D., \& Motowidlo, S. J. (2001). Personality predictors of citizenship performance. International journal of selection and assessment, 9(1-2), 52-69.

Brackett, M. A., Palomera, R., Mojsa-Kaja, J., Reyes, M. R., \& Salovey, P. (2010). Emotion-regulation ability, burnout, and job satisfaction among British secondary-school teachers. Psychology in the Schools, 47(4), 406-417.

Burger, J. M., \& Caldwell, D. F. (2000). Personality, social activities, job-search behavior and interview success: Distinguishing between PANAS trait positive affect and NEO extraversion. Motivation and Emotion, 24(1), 51-62

Butler, R. (2007). Teachers' achievement goal orientations and associations with teachers' help seeking: examination of a novel approach to teacher motivation. Journal of Educational Psychology, 99(2), 241-252.

Carmona, C., Buunk, A. P., Peiró, J. M., Rodríguez, I., \& Bravo, M. J. (2006). Do social comparison and coping styles play a role in the development of burnout? Cross-sectional and longitudinal findings. Journal of Occupational and Organizational Psychology, 79(1), 85-99.

Chang, M. L. (2009). An appraisal perspective of teacher burnout: Examining the emotional work of teachers. Educational psychology review, 21(3), 193-218.

Cropanzano, R., \& Wright, T. A. (2001). When a" happy" worker is really a" productive" worker: A review and further refinement of the happy-productive worker thesis. Consulting Psychology Journal: Practice and Research, 53(3), 182-199.

Cumming, T. (2017). Early childhood educators' well-being: an updated review of the literature. Early Childhood Education, 45(5), 583-593.

Devos, C., Dupriez, V., \& Paquay, L. (2012). Does the social working environment predict beginning teachers' self-efficacy and feelings of depression? Teaching and Teacher Education, 28(2), 206217. 
Diener E. (2000), Subjective well-being: The science of happiness and a proposal for a national index, American Psycologist, 55, 34-43.

Diener, E., Heintzelman, S. J., Kushlev, K., Tay, L., Wirtz, D., Lutes, L. D., \& Oishi, S. (2017). Findings all psychologists should know from the new science on subjective well-being. Canadian Psychology/Psychologie canadienne, 58(2), 87-104.

Duckworth, A. L., Quinn, P. D., \& Seligman, M. E. (2009). Positive predictors of teacher effectiveness. The Journal of Positive Psychology, 4(6), 540-547.

Dutton, J. E., Worline, M. C., Frost, P. J., \& Lilius, J. (2006). Explaining compassion organizing. Administrative Science Quarterly, 51(1), 59-96.

Eldor, L. (2016). Work engagement: Toward a general theoretical enriching model. Human Resource Development Review, 15(3), 317-339.

Eldor, L. (2018). Public Service Sector: The Compassionate Workplace-The Effect of Compassion and Stress on Employee Engagement, Burnout, and Performance. Journal of Public Administration Research and Theory, 28(1),86-103.

Eldor, L., \& Shoshani, A. (2016). Caring relationships in school staff: Exploring the link between compassion and teacher work engagement. Teaching and Teacher Education, 59, 126-136.

Field, L. K., \& Buitendach, J. H. (2011). Happiness, work engagement and organisational commitment of support staff at a tertiary education institution in South Africa. SA Journal of Industrial Psychology, 37(1), 01-10.

Fiorilli, C., De Stasio, S., Benevene, P., lezzi, F. D., Pepe, A., \& Albanese, 0. (2015). COPENHAGEN BURNOUT INVENTORY (CBI): A VALIDATION STUDY IN AN ITALIAN TEACHER GROUP. TPM: Testing, Psychometrics, Methodology in Applied Psychology, 22(4), 537-551.

Fredrickson, B. L. (2001). The role of positive emotions in positive psychology: The broaden-andbuild theory of positive emotions. American psychologist, 56(3), 218-226.

Fredrickson, B. L. (2003). The value of positive emotions: The emerging science of positive psychology is coming to understand why it's good to feel good. American scientist, 91(4), 330335.

Fredrickson, B. L. (2013). Positive emotions broaden and build. In E. A. Plant \& P. G. Devine (Ed.), Advances in experimental social psychology, Vol. 47. San Diego, CA: Academic Press.

Fredrickson, B. L., \& Joiner, T. (2002). Positive emotions trigger upward spirals toward emotional well-being. Psychological science, 13(2), 172-175.

Fredrickson, B. L., \& Losada, M. F. (2005). Positive affect and the complex dynamics of human flourishing. American psychologist, 60(7), 678-686.

Gwede, C. K., Johnson, D. J., Roberts, C., \& Cantor, A. B. (2005). Burnout in clinical research coordinators in the United States. Oncology Nursing Forum, 32(6), 1123-1130

Fredrickson, B. L., Tugade, M. M., Waugh, C. E., \& Larkin, G. R. (2003). What good are positive emotions in crisis? A prospective study of resilience and emotions following the terrorist attacks on the United States on September 11th, 2001. Journal of personality and social psychology, 84(2), 365-376.

Hakanen, J. J., Bakker, A. B., \& Schaufeli, W. B. (2006). Burnout and work engagement among teachers. Journal of school psychology, 43(6), 495-513.

Halbesleben, J.R.B. (2010). A meta-analysis of work engagement: Relationships with burnout, demands, resources and consequences. In A.B. Bakker \& M.P. Leiter (Ed.), Work engagement: Recent developments in theory and research (pp. 102-117). New York: Psychology Press. 
Hargreaves, A. (2000). Mixed emotions: Teachers' perceptions of their interactions with students. Teaching and teacher education, 16(8), 811-826.

Hoffman, M. L. (2000). Empathy and moral development: Implications for caring and justice. New York, NY: Cambridge University Press.

Hoy, A. W., \& Spero, R. B. (2005). Changes in teacher efficacy during the early years of teaching: A comparison of four measures. Teaching and teacher education, 21(4), 343-356.

Huynh, J. Y., Xanthopoulou, D., \& Winefield, A. H. (2014). The job demands-resources model in emergency service volunteers: Examining the mediating roles of exhaustion, work engagement and organizational connectedness. Work \& Stress, 28(3), 305-322.

Hur, E., Jeon, L., \& Buettner, C. K. (2016). Preschool teachers' childcentred beliefs: Direct and indirect associations with work climate and job-related wellbeing. Child Youth Care Forum, 45(3), 451-465.

Hwang, J. Y., Plante, T., \& Lackey, K. (2008). The development of the Santa Clara brief compassion scale: An abbreviation of Sprecher and Fehr's compassionate love scale. Pastoral Psychology, 56(4), 421-428.

Ilies, R., Scott, B. A., \& Judge, T. A. (2006). The interactive effects of personal traits and experienced states on intraindividual patterns of citizenship behavior. Academy of Management Journal, 49(3), 561-575.

Iverson, R. D., Olekalns, M., \& Erwin, P. J. (1998). Affectivity, organizational stressors, and absenteeism: A causal model of burnout and its consequences. Journal of Vocational behavior, 52(1), 1-23.

Järvelä, S., Volet, S., \& Järvenoja, H. (2010). Research on motivation in collaborative learning: Moving beyond the cognitive-situative divide and combining individual and social processes. Educational psychologist, 45(1), 15-27.

Jennings, P. A., \& Greenberg, M. T. (2009). The prosocial classroom: Teacher social and emotional competence in relation to student and classroom outcomes. Review of educational research, 79(1), 491-525.

Kahn, J. H., Schneider, K. T., Jenkins-Henkelman, T. M., \& Moyle, L. L. (2006). Emotional social support and job burnout among high-school teachers: is it all due to dispositional affectivity?. Journal of Organizational Behavior, 27(6), 793-807.

Kahn, J. H., \& Scott, N. A. (1997). Predictors of research productivity and science-related career goals among counseling psychology doctoral students. The Counseling Psychologist, 25(1), 3867.

Klusmann, U., Kunter, M., Trautwein, U., Ludtke, 0., \& Baumert, J. (2008). Engagement and emotional exhaustion in teachers: does school context make a difference. Health and Wellbeing, $57,127-151$.

Kok, B. E., Coffey, K. A., Cohn, M. A., Catalino, L. I., Vacharkulksemsuk, T., Algoe, S. B., Brantley, M., \& Fredrickson, B. L. (2013). How positive emotions build physical health: Perceived positive social connections account for the upward spiral between positive emotions and vagal tone. Psychological science, 24(7), 1123-1132.

Kokkinos, C. M. (2007). Job stressors, personality and burnout in primary school teachers. British Journal of Educational Psychology, 77(1), 229-243.

Løvgren, M. (2016). Emotional exhaustion in day-care workers. European Early Childhood Education Research Journal, 24(1), 157-167. 
Lazarus, R. S. (1993). Coping theory and research: Past, present, and future. Psychosomatic Medicine, 55, 2324-2347.

Lee, K., \& Allen, N. J. (2002). Organizational citizenship behavior and workplace deviance: The role of affect and cognitions. Journal of applied psychology, 87(1), 131-142.

Lilius, J. M., Worline, M. C., Maitlis, S., Kanov, J., Dutton, J. E., \& Frost, P. (2008). The contours and consequences of compassion at work. Journal of Organizational Behavior, 29(2), 193-218.

Linnenbrink-Garcia, L., Rogat, T. K., \& Koskey, K. L. (2011). Affect and engagement during small group instruction. Contemporary Educational Psychology, 36(1), 13-24.

Lucas, R. E. (2007). Adaptation and the set-point model of subjective well-being: Does happiness change after major life events?. Current Directions in Psychological Science, 16(2), 75-79.

Luhmann, M., Hofmann, W., Eid, M., \& Lucas, R. E. (2012). Subjective well-being and adaptation to life events: a meta-analysis. Journal of personality and social psychology, 102(3), 592-615.

Lyubomirsky, S. (2001). Why are some people happier than others? The role of cognitive and motivational processes in well-being. American psychologist, 56(3), 239-249.

Lyubomirsky, S., \& Lepper, H. S. (1999). A measure of subjective happiness: Preliminary reliability and construct validation. Social indicators research, 46(2), 137-155.

Lyubomirsky, S., King, L., \& Diener, E. (2005). The benefits of frequent positive affect: Does happiness lead to success?. Psychological bulletin, 131(6), 803-855.

Maslach, C. (1999). Progress in Understanding Teacher Burnout. In Vendenberghe R. \& Huberman A. M. (Ed.), Understanding and Preventing Teacher Burnout (pp. 211-222 ) New York: Cambridge University Press.

Mason, V. M., Leslie, G., Clark, K., Lyons, P., Walke, E., Butler, C., \& Griffin, M. (2014). Compassion fatigue, moral distress, and work engagement in surgical intensive care unit trauma nurses: a pilot study. Dimensions of Critical Care Nursing, 33(4), 215-225.

Mauno, S., Ruokolainen, M., Kinnunen, U., \& De Bloom, J. (2016). Emotional labour and work engagement among nurses: examining perceived compassion, leadership and work ethic as stress buffers. Journal of advanced nursing, 72(5), 1169-1181.

Miles, D. E., Borman, W. E., Spector, P. E., \& Fox, S. (2002). Building an integrative model of extra role work behaviors: A comparison of counterproductive work behavior with organizational citizenship behavior. International Journal of Selection and Assessment, 10(1-2), 51-57.

Morgeson, F. P., \& Humphrey, S. E. (2006). The Work Design Questionnaire (WDQ): developing and validating a comprehensive measure for assessing job design and the nature of work. Journal of applied psychology, 91(6), 1321-1339.

0'Brien, P. J. (2006). Creating compassion and connection in the work place. Journal of Systemic Therapies, 25(1), 16-36.

Parker, P. D., Martin, A. J., Colmar, S., \& Liem, G. A. (2012). Teachers' workplace well-being: Exploring a process model of goal orientation, coping behavior, engagement, and burnout. Teaching and Teacher Education, 28(4), 503-513.

Peeters, M. A., \& Rutte, C. G. (2005). Time management behavior as a moderator for the job demandcontrol interaction. Journal of occupational health psychology, 10(1), 64-75.

Pietarinen, J., Pyhältö, K., Soini, T., \& Salmela-Aro, K. (2013). Reducing teacher burnout: A sociocontextual approach. Teaching and Teacher Education, 35, 62-72.

Pyhältö, K., Pietarinen, J., \& Salmela-Aro, K. (2011). Teacher-working-environment fit as a framework for burnout experienced by Finnish teachers. Teaching and Teacher Education, 27(7), 1101-1110. 
Pomaki, G., DeLongis, A., Frey, D., Short, K., \& Woehrle, T. (2010). When the going gets tough: Direct, buffering and indirect effects of social support on turnover intention. Teaching and Teacher Education, 26(6), 1340-1346.

Pillay, H., Goddard, R., \& Wilss, L. (2005). Well-Being, Burnout and Competence: Implications for Teachers. Australian Journal of Teacher Education, 30(2), 21-31.

Rodríguez-Muñoz, A., Sanz-Vergel, A. I., Demerouti, E., \& Bakker, A. B. (2014). Engaged at work and happy at home: A spillover-Crossover model. Journal of Happiness Studies, 15(2), 271-283.

Saarni C. (1999), The development of emotional competence, Guilford Press, New York.

Salanova, M., \& Schaufeli, W. (2009). El engagement en el trabajo: cuando el trabajo se convierte en pasión. Alianza editorial.

Salmela-Aro, K. (2009). Personal goals and well-being during critical life transitions: the 4 C's e channeling, choice, co-agency and compensation. Advances in Life Course Research, 14, 6373.

Salmela-Aro, K., Tolvanen, A., \& Nurmi, J. E. (2011). Social strategies during university studies predict early career work burnout and engagement: 18-year longitudinal study. Journal of Vocational Behavior, 79(1), 145-157.

Schaufeli, W. B., \& Bakker, A. B. (2004). Job demands, job resources, and their relationship with burnout and engagement: A multi-sample study. Journal of Organizational Behavior, 25(3), 293315.

Schaufeli, W. B., Bakker, A. B., \& Salanova, M. (2006). The measurement of work engagement with a short questionnaire: A cross-national study. Educational and psychological measurement, 66(4), 701-716.

Schreyer, I., \& Krause, M. (2016). Pedagogical staff in children's day care centres in Germany-links between working conditions, job satisfaction, commitment and work-related stress. Early Years, 36(2), 132-147.

Sharplin, E., O'Neill, M., \& Chapman, A. (2011). Coping strategies for adaptation to new teacher appointments: Intervention for retention. Teaching and Teacher Education, 27(1), 136-146.

Skaalvik, E. M., \& Skaalvik, S. (2011). Teacher job satisfaction and motivation to leave the teaching profession: Relations with school context, feeling of belonging, and emotional exhaustion. Teaching and teacher education, 27(6), 1029-1038.

Soini, T., Pyhältö, K., \& Pietarinen, J. (2010). Pedagogical well-being: reflecting learning and wellbeing in teachers' work. Teachers and Teaching: theory and practice, 16(6), 735-751.

Sprecher, S., \& Fehr, B. (2005). Compassionate love for close others and humanity. Journal of Social and Personal Relationships, 22(5), 629-651.

Staw, B. M., \& Barsade, S. G. (1993). Affect and managerial performance: A test of the sadder-butwiser vs. happier-and-smarter hypotheses. Administrative Science Quarterly, 38(2), 304-331.

Staw, B. M., Sutton, R. I., \& Pelled, L. H. (1994). Employee positive emotion and favorable outcomes at the workplace. Organization Science, 5(1), 51-71.

Stoeber, J., \& Rennert, D. (2008). Perfectionism in school teachers: Relations with stress appraisals, coping styles, and burnout. Anxiety, stress, and coping, 21(1), 37-53.

Sutton, R. E., \& Wheatley, K. F. (2003). Teachers' emotions and teaching: A review of the literature and directions for future research. Educational psychology review, 15(4), 327-358.

Tehan, M., \& Robinson, P. (2009). Leading the way: Compassion in the workplace. IIIness, Crisis \& Loss, 17(2), 93-111. 
Tikkanen, L., Pyhältö, K., Pietarinen, J., \& Soini, T. (2017). Interrelations between principals' risk of burnout profiles and proactive self-regulation strategies. Social Psychology of Education, 20(2), 259-274.

Thoresen, C. J., Kaplan, S. A., Barsky, A. P., Warren, C. R., \& de Chermont, K. (2003). The affective underpinnings of job perceptions and attitudes: a meta-analytic review and integration.

Tremblay, M. A., \& Messervey, D. (2011). The Job Demands-Resources model: Further evidence for the buffering effect of personal resources. SA Journal of Industrial Psychology, 37(2), 10-19.

Tugade, M. M., \& Fredrickson, B. L. (2004). Resilient individuals use positive emotions to bounce back from negative emotional experiences. Journal of personality and social psychology, 86(2), 320.

Underwood, R. A. (2009). U.S. Patent No. 7,491,200. Washington, DC: U.S. Patent and Trademark Office.

Warr, P. (2007). Work, Happiness, and Unhappiness. Mahwah, NJ: Lawrence Erlbaum.

Watson, D., \& Clark, L. A. (1999). The PANAS-X: Manual for the positive and negative affect schedule-expanded form.

Watson, D., Clark, L. A., \& Carey, G. (1988). Positive and negative affectivity and their relation to anxiety and depressive disorders. Journal of abnormal psychology, 97(3), 346.

Watson, D., Clark, L. A., \& Tellegen, A. (1988). Development and validation of brief measures of positive and negative affect: the PANAS scales. Journal of personality and social psychology, 54(6), 1063.

Watson, D., \& Tellegen, A. (1985). Toward a consensual structure of mood. Psychological bulletin, 98(2), 219.

Weiss, H. M., \& Cropanzano, R. (1996). Affective Events Theory: A theoretical discussion of the structure, causes and consequences of affective experiences at work. In B. M. Staw \& L. L. Cummings (Ed.), Research in organizational behavior: An annual series of analytical essays and critical reviews, Vol. 18, ( pp. 1-74). US: Elsevier Science/JAI Press.

Xanthopoulou, D., Bakker, A. B., Demerouti, E., \& Schaufeli, W. B. (2007). The role of personal resources in the job demands-resources model. International journal of stress management, 14(2), 121.

Xanthopoulou, D., Bakker, A. B., Demerouti, E., \& Schaufeli, W. B. (2009). Reciprocal relationships between job resources, personal resources, and work engagement. Journal of Vocational behavior, 74(3), 235-244. 

